\title{
Comparative evaluation of 64-slice CT angiography and digital subtraction angiography in assessing the cervicocranial vasculature
}

\author{
Randolf Klingebiel' \\ Max Kentenich ${ }^{3}$ \\ Hans-Christian Bauknecht ${ }^{3}$ \\ Florian Masuhr ${ }^{2}$ \\ Eberhard Siebert ${ }^{\prime}$ \\ Markus Busch ${ }^{2}$ \\ Georg Bohner' \\ 'Department of Neuroradiology, \\ ${ }^{2}$ Department of Neurology, \\ ${ }^{3}$ Department of Radiology, Charité \\ Universitary Medicine Berlin, \\ Germany
}

\begin{abstract}
Background: Noninvasive 64-slice computed tomography angiography (64-MSCTA) closely approximates conventional catheter angiography (DSA) in terms of detail resolution.

Objective: Retrospective evaluation of cervicocranial (cc) 64-MSCTA in comparison with DSA in patients with presumptive cc vascular disorders.

Material and methods: Twenty-four 64-MSCTA studies (32 mm detector width, slice thickness $0.5 \mathrm{~mm}, 120 \mathrm{kv}, 150 \mathrm{mAs}$, pitch 0.75 ) of patients with presumptive cc vascular pathology (13 men, 11 women, mean age $38.3 \pm 11.3$ yrs, range 19-54 yrs) were assessed in comparison with DSA studies without abnormal findings in age-matched patients (11 men, 13 women, mean age $39.7 \pm 11.9$ yrs, range $18-54$ yrs). Study readings were performed in a blinded manner by two neuroradiologists with respect to image quality and assessibility of various cc vascular segments by using a five-point scale. Radiation exposure was calculated for 64-MSCTA.

Results: Each reader assessed 384/528 different vessel segments (64-MSCTA/DSA). Superior image quality was attributed to DSA with respect to the C1 ICA-C6 ICA, A3 ACA, and P3 PCA segments as well the AICA and SCA. 64-MSCTA was scored superior for C7 ICA and V4 VA segments. A significantly increased number of nonassessable V2- and V3 VA segments in DSA studies was noted. The effective dose for 64-MSCTA amounted to $2.2 \mathrm{mSv}$.

Conclusions: 64-MSCTA provides near-equivalent diagnostic information of the cc vasculature as compared with DSA. According to our results, DSA should be considered primarily when peripheral vessels (A3/P3) or ICA segments close to the skull base (C2-5) are of interest, such as in primary angiitis or stenoocclusive ICA disease, respectively.
\end{abstract}

Keywords: CT-angiography, CNS, angiography

\section{Introduction}

Computed tomography angiography (CTA) has increasingly gained attention since the introduction of multislice CT (MSCT). CTA using multislice scanners including the latest generation of 64-slice scanners (64-MSCTA) permits rapid and comprehensive assessment of the cervico-cranial vasculature, thus becoming a valuable tool in stroke patients (Klingebiel et al 2002; Schuknecht 2004).

Recent studies underline the suitability of MSCTA for assessing various cervico-cranial vascular pathologies such as carotid artery stenosis (Bartlett et al 2006; Silvennoinen et al 2007), carotid and vertebral artery injuries (Utter et al 2006) and carotid artery dissection (Elijovich et al 2006), amongst others.

For X-ray-based, cervicocranial (cc) vessel assessment, several issues merge in when a decision has to be made as to what modality is appropriate. Given that stateof-the-art multislice CTA is available as well as a neurorangiography suite to perform a conventional catheter angiography (DSA) and no immediate vascular intervention is considered, the required detail resolution, invasiveness, radiation exposure, and cost efficiency will all affect the choice of the imaging modality. 
The purpose of our study was to define differences in image quality at various segmental levels of the cc vasculature in order to elucidate criteria for decision-making between 64-MSCTA and DSA in patients with presumptive cc vascular insufficiency.

\section{Patients and methods}

Twenty-four 64-MSCTA studies (13 men, 11 women, mean age $38.3 \pm 11.3 \mathrm{yrs}$, range $19-54 \mathrm{yrs}$ ) were retrospectively assessed in comparison to DSA studies in an age-matched group of patients ( $11 \mathrm{men}, 13$ women, mean age $39.7 \pm 11.9 \mathrm{yrs}$, range $18-54 \mathrm{yrs}$ ), all studies previously reported as being without abnormal findings. All patients had been referred by the departments of neurology and/or internal medicine because of presumptive pathology of the cc vasculature.

MSCTA was performed using a CT scanner equipped with 64 detector rows (Aquilion 64, Toshiba Medical Systems Europe, The Netherlands). The following parameters were applied: $0.5 / 0.3 \mathrm{~mm}$ collimation/increment, $120 \mathrm{kv}, 150 \mathrm{mAs}$, pitch $0.75,240 \mathrm{~mm}$ FOV, 512 matrix). In order to avoid inproper bolus timing due to (infra-) bifurcational internal and/or common carotid artery pathology, the scan start was manually initiated whenever contrast enhancement within the suprabifurcational internal carotid artery (ICA) was detected by the radiologist during a low-dose dynamic scan at the level of the third to fourth cervical vertebral body. Iodinated, nonionic contrast medium (Ultravist 370, Schering, Germany) was applied by a power injector (Nemoto Kyorindo, Tokyo, Japan) with a flow rate of $4 \mathrm{ml} / \mathrm{s}$ and a total volume of $80 \mathrm{ml}$, chased by $20 \mathrm{ml}$ of $0.9 \%$ saline using the same flow rate.

Digital subtraction angiography of the cc vasculature in standard planes was achieved in an angiography suite (Angiostar, Siemens, Germany) subsequent to inguinal, transfemoral puncture by repeated, selective injection of iodinated contrast medium (Ultravist 300, Bayer Vital, Germany) through a simple curve end-hole catheter into the common and internal carotid arteries (ICA) as well as the vertebral arteries (VA). DSA images were exclusively assessed by hardcopy readings.

In preparation of the study readings, all MSCTA data were transferred from the archive to a workstation (Vitrea 2, Version 3.3, Vital Images Europe B.V., The Netherlands) via internal network connections, providing 3D postprocessing options, ie, multiplanar image reformatting (MPR) and maximum intensity projections (MIP).

Study readings were performed in a blinded manner by two neuroradiologists with respect to image quality and assessibility by using a five-point scale. The readers interactively generated standardized $3 \mathrm{D}$ views based on maximum intensity projections (MIP). In addition cine view assessment of cross-sectional source images as well as multiplanar reformatted images (MPR) were used by the readers. The scale was applied to the following cc vessels: internal carotid artery (ICA; segments $\mathrm{C} 1$ to $\mathrm{C} 7$ ), anterior cerebral artery (ACA; segments $\mathrm{A} 1$ to A3), middle cerebral artery (MCA; segments M1 to M3), posterior cerebral artery (PCA; segments $\mathrm{P} 1$ to $\mathrm{P} 3$ ), vertebral artery (VA; segments V2 to V4), anterior and posterior inferior cerebellar artery (AICA and PICA), basilar artery (BA), and superior cerebellar artery (SCA). The vascular segments were defined according to the literature (Osborn 1998) and an evaluation spread sheet (Excel 2000, Microsoft Corporation, Redmond, WA) was used with implemented links to schematic drawings of the cc vasculature in order to assure uniform nomenclature for all 23 (64-MSCTA) and 27 (DSA) vessel segments, respectively.

Image quality study results were statistically evaluated by using a Mann-Whitney Rank Sum test. Weighted kappa statistics were used for determining interobserver variability and interpreted according to the literature (Landis et al 1977).

Differences between CTA and DSA in the number of segments rated as nonassessable were statistically adressed by applying a McNemar test.

For test performances, a commercially available software tool (SigmaStat 3.1, Systat Software, Inc., Richmond, WA) was used.

Radiation exposure was defined for 64-MSCTA by calculating the parameters Effective dose (ED) and effective, weighted CT dose index (CTDIw,eff), using a commercially available software module (CT-EXPO, release 1.3; G. Stamm, Medical School Hannover, Dept. of Experimental Radiology, Hannover, Germany).

\section{Results}

Each reader assessed 384 and 528 different vessel segments for 64-MSCTA and DSA respectively. The lower number of segments assessed by 64-MSTCA as opposed to DSA is explained by selective bilateral readings of V2 VA to V4 VA and PICA segments as well as bilateral AICA and SCA readings in DSA studies. These additional DSA readings were performed in order to avoid a rating bias caused by retrograde filling differences in studies with only unilateral VA intubation as well as a projection-dependant impact on image quality in subtle vascular structures such as PICA, AICA, and SCA. In these cases only the score of the intubated vessel (VA, PICA) was included for statistical purposes. The mean value of bilateral DSA readings was used for AICA and SCA statistics. 
For a detailed result and overview of image quality assessment, the reader is referred to Figures 1 and 2. In summary, superior image quality was attributed by the readers to DSA with respect to the C1 ICA-C6 ICA segments, the A3 ACA, P3 PCA, as well as the AICA and SCA.

64-MSCTA was scored superior with respect to C7 ICA and V4 VA segments.

Reasons for image quality impairment in 64-MSCTA, as noted by the readers, were superposition by osseous structures $(n=17)$ or neighboring vessels ( 8 vessel segments), incomplete vessel visualization, ie, the full vessel length was not included within the scan area $(n=15)$ as well as image artifacts caused by dental implants $(n=4)$ (Figure 3).

Vessel segments most often affected in 64-MSCTA were C1 ICA, A3 ACA, P3 PCA, V2 VA, and the AICA.

In DSA studies, the following factors were mentioned on the evaluation spreadsheet as impairing segmental vessel scores: visualization in only one plane $(n=8)$, motion artifacts affecting image subtraction $(n=4)$, incomplete visualization of full vessel segment length $(n=2)$, solely unilateral visualization of paired vessels $(n=2)$, as well as various technical reasons (blurring of image borders and peripheral vessel segments, unsuitable projection for the target vessel, etc.; $n=5$ ).
In five patients, instead of selective ICA intubation, the anterior circulation had been addressed by CCA injection with subsequent superposition of ICA and ECA branches. This also affected ACA and MCA vessel scores in these patients.

With respect to the V2- and V3 VA segment, there was a significant increase in the number of nonassessable segments in DSA studies as compared with 64-MSCTA (McNemar test, $\mathrm{p}<0.001)$.

Effective dose (ED) and CTDIw,eff values for 64-MSCTA amounted to $2.2 \mathrm{mSv}$ and $44.3 \mathrm{mGy}$, respectively.

The weighted kappa value for overall interobserver agreement amounted to $\mathrm{k}=0.39$, indicating fair to moderate agreement.

The scan protocol applied provided isotropic high resolution voxels of about $0.4 \mathrm{~mm}$ size.

The pixel size of our singleplane Angio lab (Angiostar, Siemens, Germany) amounted to about $0.2 \mathrm{~mm}$.

\section{Discussion}

Since the introduction of the first multislice scanner generation in 1999 (Hu 1999), an ongoing debate has arisen about the borderline between CTA and DSA for diagnostic purposes. With respect to DSA, the invasive character of this technique represents an important difference as compared with CTA. The overall incidence of neurological deficits due to procedural

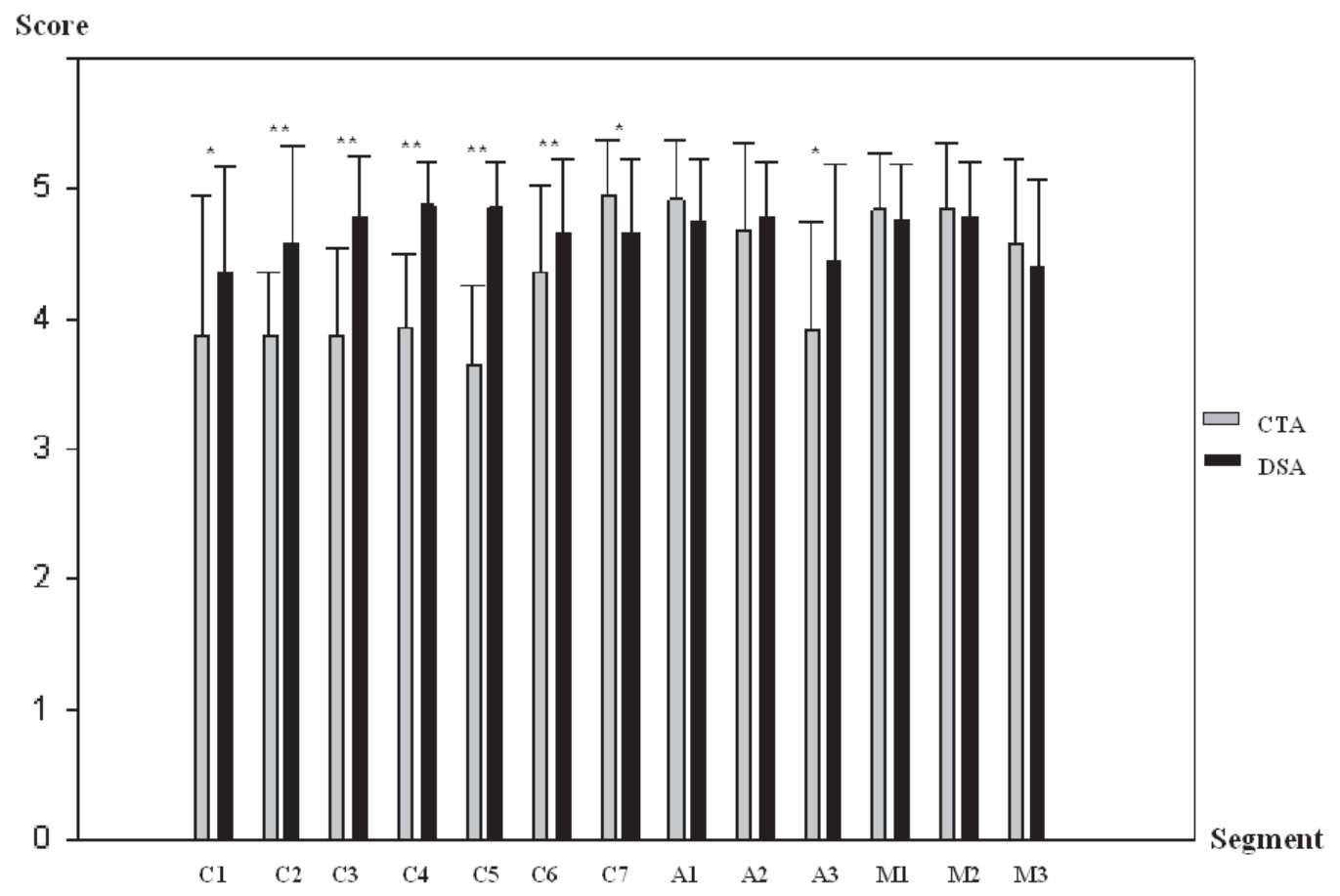

Figure I Results of the Image quality readings, anterior circulation by using a five-point scale ( $I$ = insufficient, $5=$ excellent image quality). Notes: ${ }^{*} \mathrm{p}<0.05,{ }^{* *} \mathrm{p}<0.001$. 


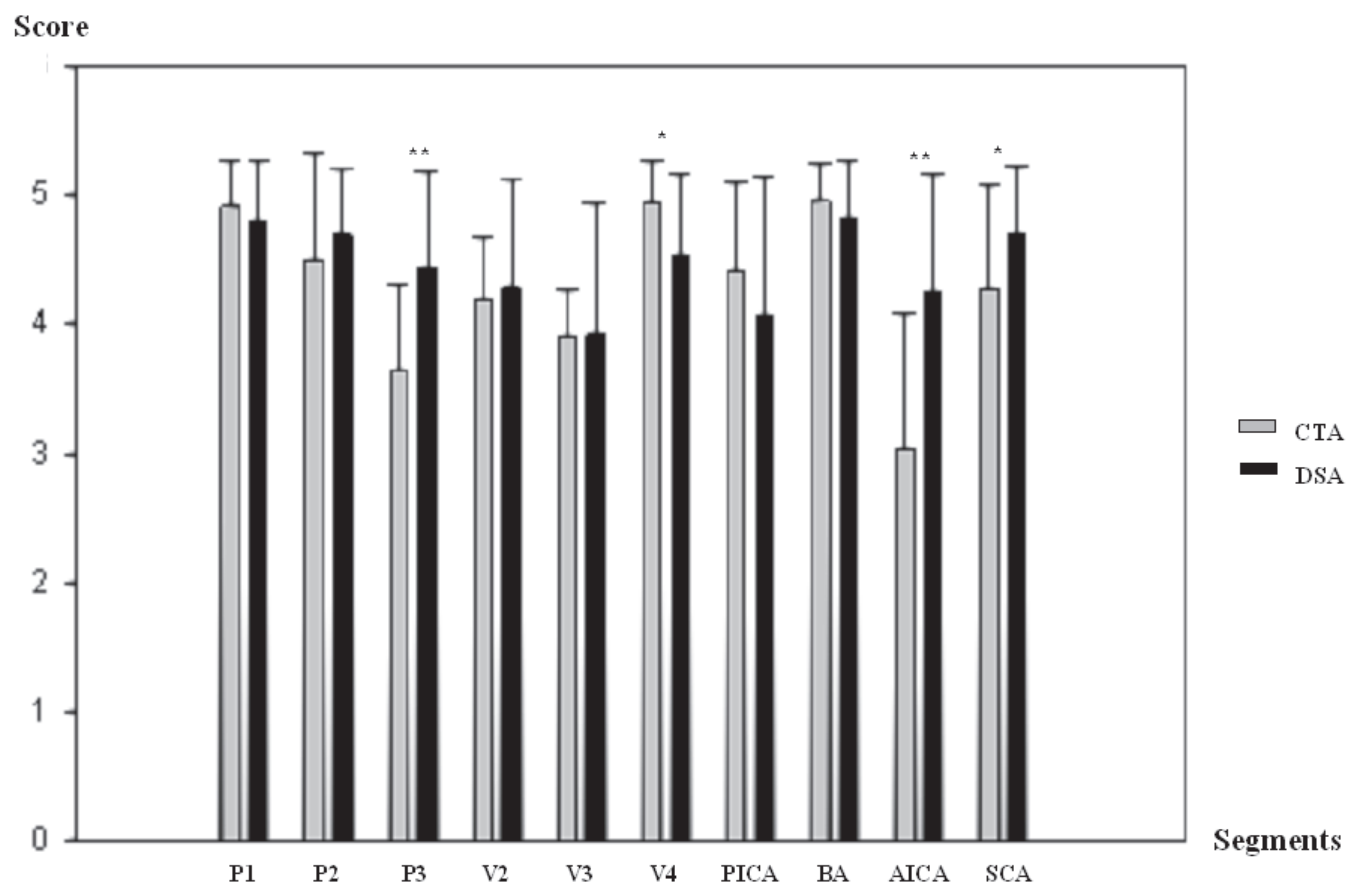

Figure $\mathbf{2}$ Results of the Image quality readings, posterior circulation (same score as in Figure I). Note: ${ }^{* *} \mathrm{p}<0.001$.

complications during DSA has been reported to amount to $1 \%$ to $2.6 \%$, with an incidence of persistent deficits following cerebral angiography of about 0.1 to $0.5 \%$ (Heiserman et al 1994; Leffers et al 2000; Kaufmann et al 2007). Yet, nonneurological complications were encountered in $14.7 \%$ in the study of Leffers and colleagues (2000) and clinically silent embolisms were encountered in up to $44 \%$ of patients undergoing DSA when vascular risk factors were present (Bendszus et al 1999). The duration of the procedure as well as the investigator experience are factors that influence the complication rate (Kaufmann et al 2007). Anatomic variants of the aortic arch such as a type II arch which is encountered in up to one third of patients may protract the DSA imaging procedure, resulting in an increased procedural risk as well as impaired image quality if selective internal carotid artery intubation is not achieved.

In consequence, DSA should not be performed if a less invasive imaging technique is available.

Whether or not DSA is required depends upon the diagnostic performance of the competing technique, ie, CTA in our study. The diagnostic performance itself largely depends on the image quality provided at various levels of the cc vasculature.

Whereas DSA, CTA, MRA, and color-coded Doppler sonography all have been reported as suitable for assessing steno-occlusive at the level of the suprabifurcational ICA, peripheral vascular segments are only appropriately visualized intracranially by high-resolution imaging.
M1 MCA diameters have been reported to range from 2.5 to $4 \mathrm{~mm}$ (Pai et al 2005), whereras peripheral segments (M2-3, pericallosal artery) are in the 1 to $2 \mathrm{~mm}$ size range and AICA diameters of about $1 \mathrm{~mm}$ have been described (Villablanca et al 2006, 2007).

In order to rule out so-called partial volume artifacts and to comprehensively assess these vessels, the size of the smallest image volume elements (voxel) has to be in the submillimeter range. This prerequisite is fulfilled by CTA and DSA as well. Nevertheless, 64-MSCTA, the latest and best performing MSCT scanner generation, yielded inferior image quality with regard to A3 ACA, P3 PCA, and AICA segments as compared with DSA.

Villablanca and colleagues (2007) recently described the smallest arterial size reliably detected with MSCTA as being $0.7 \mathrm{~mm}$ (4-16 slice Scanner) versus $0.4 \mathrm{~mm}$ for DSA. In our study, a scanner with the smallest collimation currently available $(0.5 \mathrm{~mm})$ was used, allowing for an in-plane resolution of $0.5 \mathrm{~mm}$ and a voxel size of $0.5 \times 0.5 \times 0.4 \mathrm{~mm}$, thus delivering isotropic voxels of a size that closely approximates DSA spatial resolution in the study of Villablanca and co-workers.

Most probably, image quality differences are explained by other factors than calculatory spatial resolution: vesselto-background ratio and superposition by other vascular structures in image reconstructions (eg, basal vein of 


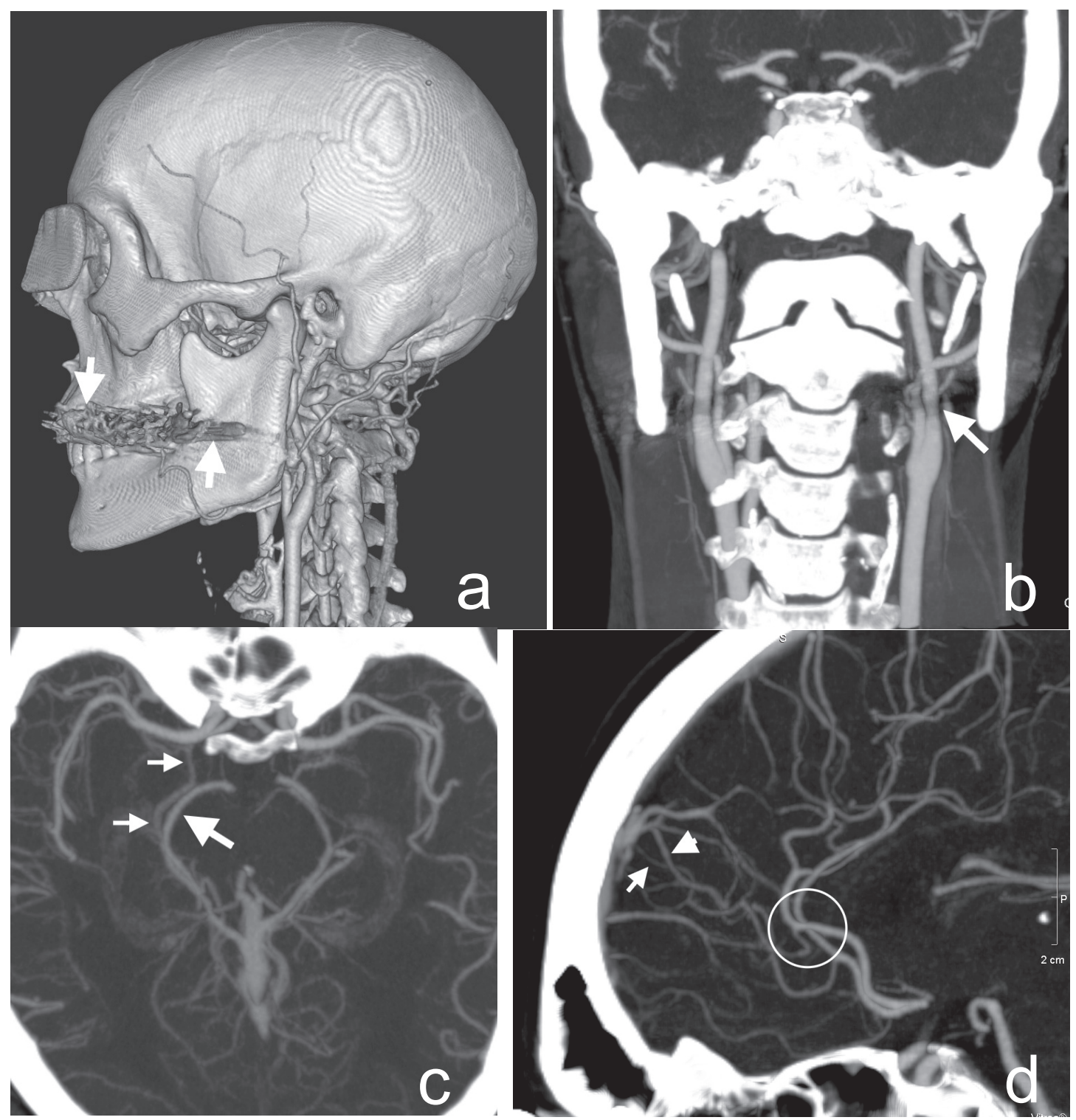

Figure 3 Methodological limitations of 64-MSCTA, resulting in impaired image quality scores. (a-b) Dental implant artifacts (arrows) are depicted (a, 3D-reconstruction, lateral view), causing II ICA pseudostenosis (arrow in b, coronal MIP). (c) Axial MIP reconstruction. Superimposed basal vein of Rosenthal (arrow) on to the right-sided P2/3 PCA (small arrows). (d) Overlay of interhemispheric veins (arrowhead) on subtle branches of the marginal callosal artery (arrow). In addition, MIP reconstructions suffer from the pitfall of pseudo-stenosis at vessel crosspoints (circle).

Abbreviations: ICA, internal carotid artery; MIP, maximum intensity projections; 64-MSCTA, 64-slice computed tomography angiography; PCA, posterior cerebral artery.

Rosenthal). Vessel-to-background ratio is enhanced in DSA due to the subtraction technique, eliminating undesirable image information. DSA allows selective vessel intubation with less dilution effects and improved signal-to-noise ratio than expected when using intravenous access in CTA.

Thus, presumptive vascular pathology with involvement of small peripheral vessels in the range of $\leq 1 \mathrm{~mm}$ outer diameter should primarily be addressed by DSA, if available. This is especially true when primary angiitis of the CNS (PACNS) is considered, as rapid treatment is possible and required in order to prevent further parenchymal damage. Yet, even when using DSA, a sensitivity as low as about $30 \%$ has been decribed when DSA is not supported by other diagnostic tests (CSF tap, etc) (Vollmer et al 1993).

Magnetic resonance angiography (MRA), a different, widely used noninvasive imaging technique for assessing intracranial vasculature is commonly performed using 3-dimensional (3D) time-of-flight (3D TOF) sequences. Although improved spatial resolution with voxel sizes $<1 \mathrm{~mm}^{3}$ has been reported for 3D TOF MRA (Schellinger et al 2007), superior in-plane resolution defined by pixel sizes in the 
0.2 to $0.4 \mathrm{~mm}$ range still speaks in favor of CTA and DSA when small vessel disease has to be addressed.

Other segments with impaired image quality in our study were attributable to the ICA in close proximity to the skull base. In a recent study Tomandl and colleagues (2006) showed, that 7 out of 13 aneurysms, located at the skull base, could not be detected unless a bone subtraction algorithm was applied. This is in agreement with our clinical experience based on several thousand MSCT angiograms throughout the past seven years, using 4-, 16-, 32-, and 64slice scanners.

Especially small pseudoaneurysms and/or circumscribed vessel wall dissections might be overlooked if not thoroughly sought at the skull base level.

Thus, in patients with presumptive vessel dissection and primarily negative MSCT angiograms, MSTCA should be reevaluated and/or followed at short term by DSA.

Radiation exposure (RE) is a matter of concern whenever $\mathrm{X}$-ray based imaging techniques are applied. RE is a variable that depends on the DSA procedure itself (2-, 3-, or 4 vessel angiography), vascular anatomy (elongation, anatomic variants), investigator experience and angiography suite used. Moreover, various exposure parameters are used, such as Effective Dose (ED), CTDI (for MSCTA), organ dose (for example lens dose), etc. A typical four vessel angiogram was found to result in a patient $\mathrm{ED}$ of $3.6 \mathrm{mSv}$ (Marshall et al 1995) which is within the range for multislice cc CTA (2,2 to $4,3 \mathrm{mSv}$ for 4 - and 64-slice CTA), as measured in our institution.

Procedural time requirements clearly speak in favor of 64-MSCTA as data acquisition $(<10 \mathrm{sec})$, data reconstruction, data transfer via internal network connections, and image postprocessing should not add up to more than fifteen minutes when performed by an experienced investigator, rendering this technique especially suitable for assessing emergency patients.

The retrospective character and interindividual comparison of image quality are shortcomings of our study.

Yet, image quality is a parameter that might easily be obscured by any kind of vascular pathology thus disabling meaningful statistics, if not huge cohorts with only one type of vessel disease and homogenous disease extent throughout the affected patient group are present. These prerequisites are almost impossible to achieve even in multicenter studies.

For these reasons, we decided to assess image quality in angiographically healthy, age-matched subjects. Repeated and short term eposure to radiation and/or an invasive angiographic procedure for study purposes most probably will not be accepted by the relevant ethics committee for obvious reasons.

Differences in comprehensiveness of cc vasculature assessment that spoke in favor of 64-MSCTA could have been avoided by using a prospective study designs including 4-vessel DSA. Yet, such a study design also carries risks for the patient as it is known that the procedural complication rate increases with the number of vessels catheterized (Leffers et al 2000).

Another shortcoming of our study is the lack of postprocessing functionality of DSA readings as opposed to CTA readings, all of which were interactively performed using a workstation.

In our study, weighted kappa statistics indicated only fair to moderate interobserver agreement. If precisely defined pathologies such as grading of stenotic vessel segments are evaluated, there is a clear cutoff between ordered rating levels. The differentiation between good and excellent image quality, for example, in angiographic studies without abnormal findings represents a rather subjective statement of the reader. Thus, instead of a five-point scale, as applied herein, rating categories such as excellent, sufficient, and insufficient might have been more appropriate.

\section{Conclusion}

64-MSCTA provides near-equivalent diagnostic visualization of the cc vasculature as compared with DSA. According to our results, DSA should be considered primarily when peripheral vessels $(\mathrm{A} 3 / \mathrm{P} 3)$ or ICA segments close to the skull base (C2-5), are of interest, such as in primary angiitis or stenoocclusive ICA disease, respectively.

Radiation exposure figures (effective dose) of CTA and DSA are equivalent when considering different MSCT scanner generations.

MSTCA provides more comprehensive cervicocranial vasculature information as the number of contrast-enhanced vessels is not correlated to procedural risks as in DSA.

\section{References}

Bartlett ES, Walters TD, Symons SP, et al. 2006. Quantification of carotid stenosis on CT angiography. AJNR Am J Neuroradiol, 27:13-19.

Bendszus M, Koltzenburg M, Burger R, et al. 1999. Silent embolism in diagnostic cerebral angiography and neurointerventional procedures: a prospective study. Lancet, 354:1594-7.

Elijovich L, Kazmi K, Gauvrit JY, et al. 2006. The emerging role of multidetector row CT angiography in the diagnosis of cervical arterial dissection: preliminary study. Neuroradiology, 48:606-12.

Heiserman JE, Dean BL, Hodak JA, et al. 1994. Neurologic complications of cerebral angiography. AJNR Am J Neuroradiol, 15:1401-7.

Hu H. 1999. Multi-slice helical CT: scan and reconstruction. Med Phys, 26:5-18. 
Kaufmann TJ, Huston J 3rd, Mandrekar JN, et al. 2007. Complications of diagnostic cerebral angiography: Evaluation of 19,826 consecutive patients. Radiology, 243:812-19.

Klingebiel R, Busch M, Bohner G, et al. 2002. Multi-slice CT angiography in the evaluation of patients with acute cerebrovascular disease - a promising new diagnostic tool. J Neurol, 249:43-9.

Landis JR, Koch GG. 1977. The measurement of observer agreement for categorical data. Biometrics, 33:159-74.

Leffers AM, Wagner A. 2000. Neurologic complications of cerebral angiography. A retrospective study of complication rate and patient risk factors. Acta Radiol, 41:204-10.

Marshall NW, Noble J, Faulkner K. 1995. Patient and staff dosimetry in neuroradiological procedures. Br J Radiol, 68:495-501.

Osborn AG. 1998. Diagnostic Cerebral Angiography, 2nd Edition. Philadelphia, USA: Lippincott Williams and Wilkins.

Pai SB, Varma RG, Kulkarni RN. 2005. Microsurgical anatomy of the middle cerebral artery. Neurol India, 53:186-90.

Schuknecht B. 2004. Latest techniques in head and neck CT angiography. Neuroradiology, 46:s208-13.

Silvennoinen HM, Ikonen S, Soinne L, et al. 2007. CT angiographic analysis of carotid artery stenosis: Comparison of manual assessment, semiautomatic vessel analysis, and digital subtraction angiography. AJNR Am J Neuroradiol, 28:97-103.
Schellinger PD, Richter G, Köhrmann M, et al. 2007 Noninvasive angiography (magnetic resonance and computed tomography) in the diagnosis of ischemic cerebrovascular disease. Cerebrovasc Dis, 24(Suppl 1):16-23.

Tomandl BF, Hammen T, Klotz E, et al. 2006. Bone-subtraction CT angiography for the evaluation of intracranial aneurysms. AJNR Am J Neuroradiol, 27:55-9.

Utter GH, Hollingworth W, Hallam DK, et al. 2006. Sixteen-slice CT angiography in patients with suspected blunt carotid and vertebral artery injuries. J Am Coll Surg, 203:838-8.

Villablanca JP, Nael K, Habibi R, et al. 2006. 3 T contrast-enhanced magnetic resonance angiography for evaluation of the intracranial arteries: comparison with time-of-flight magnetic resonance angiography and multislice computed tomography angiography. Invest Radiol, 41:799-05.

Villablanca JP, Rodriguez FJ, Stockman T, et al. 2007. MDCT angiography for detection and quantification of small intracranial arteries: comparison with conventional catheter angiography. AJR Am J Roentgenol, 188:593-602.

Vollmer TL, Guarnaccia J, Harrington W, et al. 1993. Idiopathic granulomatous angiitis of the central nervous system. Diagnostic challenges. Arch Neurol, 50:925-30. 
